\title{
Cranial Intraosseous Meningiomas: CT and MRI Findings
}

\author{
Erkan GOKCE ${ }^{1}$, Murat BEYHAN², Leyla $\mathrm{ACU}^{3}$ \\ ${ }^{1}$ Tokat Gaziosmanpaşa University, School of Medicine, Department of Radiology, Tokat, Turkey \\ ${ }^{2}$ Tokat State Hospital, Department of Radiology, Tokat, Turkey \\ ${ }^{3}$ Zurich University, School of Medicine, Department of Radiology, Zurich, Switzerland \\ Corresponding author: Erkan GOKCE drerkangokce@gmail.com
}

\section{ABSTRACT}

AIM: To evaluate computed tomography (CT) and magnetic resonance imaging (MRI) findings of cranial intraosseous meningiomas (IOMs).

MATERIAL and METHODS: This study included ten patients (six females and four males) with IOMs who underwent cranial CT and/ or MRI examinations during May 2009-June 2018. Lesions were classified based on the following locations: the sphenoid ridge, the calvarial convexity, other skull base bones, and the sphenoid ridge + calvarial extension. Bony extension, contour irregularity or radial bone spiculation, bony changes (hyperostotic, lytic, or mixed patterns), dural calcification, cerebral edema, and the presence of soft tissue were evaluated.

RESULTS: A total of eleven IOMs were identified in ten patients. The age of patients was $46-80$ (mean: $55.30 \pm 9.84$ ) years. Five of the lesions were located in the sphenoid ridge + calvarium, three in the sphenoid ridge, two in the skull base, and one in the calvarial convexity. Seven lesions exhibited radial bone spiculation. Moreover, nine lesions exhibited hyperostotic CT pattern; while, two exhibited a mixed pattern. Three IOMs were accompanied with dural calcification, and peritumoral edema was observed in six IOMs.

CONCLUSION: IOMs are predominantly low-grade tumors, commonly located in the periorbital area and often accompanied by soft tissue components and dural infiltrations. They most commonly lead to radial bone spiculation and bone expansion. Patients primarily have single lesions but may occasionally have multiple ones.

KEYWORDS: Computed tomography, Cranium, Intraosseous meningioma, Magnetic resonance imaging

\section{INTRODUCTION}

$\mathrm{M}$ ost meningiomas originating from the meningothelial cells are termed as primary intradural meningiomas (PIMs) and are located in the subdural space. Meningiomas lacking any dural connection are ectopic or extradural in nature and may originate from sites such as the skin, nasopharynx, or neck $(3,5,27)$. Primary extradural meningiomas (PEMs) are relatively rare lesions and account for $<2 \%$ of all meningiomas. Furthermore, intraosseous meningiomas (IOMs) are a group of PEMs and constitute approximately two-thirds of all extradural meningiomas $(5,7,12,17)$.
Over the last two decades, there has been an increase in reports on IOM in the literature (5). Rather than an increase in the actual prevalence of these lesions, this increase most probably reflects developments in diagnostic tools, such as improved histopathological and immunostaining techniques as well as improved radiographic techniques including computed tomography $(\mathrm{CT})$ and magnetic resonance imaging (MRI) (5). Although IOMs are benign and slow-growing tumors, the possibility of their malignant transformation is higher than that of PIMs $(5,17,31)$. Radiologically, they exhibit sclerotic, lytic, or mixed patterns, mimicking benign or malignant primary bone tumors or metastases $(5,12,15,31)$. 
Our study presents the CT and MRI findings of patients with IOMs.

\section{MATERIAL and METHODS}

This study was approved by our institutional ethics committee (No. 16-KAEK-048), and included ten patients who had IOMs of the 776 patients whose cranial CT and/or MRI examination reports reflected findings of meningioma using the picture archive communication system of our hospital from May 2009 to July 2018. Five patients with IOMs in their radiology reports were excluded from the study owing to the absence of histopathology results. Of the ten patients included in the study, six were females and four were males.

All cases had both CT and MRI examinations. All CT examinations were performed on 8- and 128-slice multidetector CT scanners. MRI examinations were carried out using a 1.5 T MRI machine. Lang et al. classified IOMs into three types (17). Type I tumors included lesions that were purely extracalvarial with no attachment to the bone; type II were purely calvarial, being located entirely within the bone of the skull; and type III corresponded to calvarial tumors with extracalvarial extension, i.e., a tumor that was located within the skull but also had a soft tissue component that extended extracranially. Type II and type III tumors were subdivided into skull base or convexity tumors. In our study, this classification was done according to the location of the lesions, such as those in the sphenoid ridge, the calvarial convexity, other skull base bones, and the sphenoid ridge + calvarial extension. Bony expansion, contour irregularity or radial spiculation, bony changes (hyperostotic, lytic, or mixed patterns), dural calcification, and existence of soft tissue component were evaluated. Presence of accompanying cerebral edema, degree of the edema (mild or severe), as well as the changes in the edema in follow-up examinations were studied. A dense linear structure over $100 \mathrm{HU}$ along the dural surface, next to the IOM on CT, was considered as dural calcification.

The MRI protocol included axial isotropic gradient T1weighted images (repetition time (TR): $9.18 \mathrm{~ms}$, echo time (TE): $6.56 \mathrm{~ms}$, inversion time (TI): $420 \mathrm{~ms}$, slice thickness: 1 $\mathrm{mm}$, flip angle: $12^{\circ}$, averages: 1 ), axial and sagittal fast spinecho T2-weighted images (TR: 5,856 ms, TE: $121 \mathrm{~ms}$, slice thickness: $5.5 \mathrm{~mm}$, averages: 2), coronal fluid attenuated inversion recovery (FLAIR) images (TR: 10,000 ms, TE: 90.2 $\mathrm{ms}$, Tl: 2,688 ms, slice thickness: $5.5 \mathrm{~mm}$, slice gap: $7 \mathrm{~mm}$ ), axial or coronal contrast-enhanced spin-echo (TR: 2,683 ms, TE: $17.1 \mathrm{~ms}$, TI: $903 \mathrm{~ms}$, slice thickness: $5.5 \mathrm{~mm}$, slice gap: 7 $\mathrm{mm}$ ), axial gradient-echo T1-weighted images (TR: $9.18 \mathrm{~ms}$, TE: $3.56 \mathrm{~ms}$, TI: $420 \mathrm{~ms}$, slice thickness: $1 \mathrm{~mm}$, flip angle: $12^{\circ}$, averages: 1), and B0 and B1000 diffusion-weighted images. Post-contrast imaging was performed on eight patients using intravenous administration of gadolinium-based contrast agent.

Based on the study by llica et al., dural enhancement or adjacent intracranial dural or extracranial soft tissue mass and associated parenchymal edema were investigated (12). Linear or nodular dural-based soft tissue enhancement, greater than
$3 \mathrm{~mm}$ in size, was considered as dural infiltration or mass. On MRI, presence of increased T2/FLAIR signal intensity in the adjacent brain parenchyma was considered as cerebral edema.

\section{RESULTS}

A total of 11 IOMs was observed. Incidence rate of IOMs among all the intracranial meningiomas was $1.4 \%$. Age range of the patients with IOMs was $46-80$ years (mean: $55.30 \pm 9.84$ years). They were located unilaterally in nine patients (Figures 1A-D; 2A-D; 3A-D) and bilaterally in one patient (Figure 4A-F). Follow-up periods of the patients ranged from 1 to 73 months (mean: $24.36 \pm 26.42$ months).

In terms of grading, ten of the IOMs were diagnosed as World Health Organization (WHO) grade I and one as WHO grade II. Five of the lesions were located in the sphenoid ridge + calvarium, three in the sphenoid ridge, one in the calvarial convexity, and two in the base of the skull. In terms of CT patterns of the IOMs, nine of them exhibited a hyperostotic pattern (Figures 1A-D, 4A-F), and two exhibited a mixed pattern (Figure $3 A-D$ ). Three of the IOMs had calcifications in the adjacent dura mater on CT (Figure 4A-F). Four of the lesions had edema in the adjacent cerebral parenchyma at the time of diagnosis; while, two of them developed edema during the follow-up periods (Figures 2A-D, 3A-D). Moreover, three of the lesions had severe edema and three had slight edema. Radial bone spiculation was observed in the inner or outer tabula contours of seven lesions. Based on the Lang classification, all the IOMs were classified as type III tumors. All IOMs had dural involvement, except for those in two patients who did not receive contrast agent during MRI study. Demographic characteristics and radiological findings of the patients are summarized in Table I.

Radiological examinations were planned due to orbital swelling (four cases), headache (two cases), swelling in head (two cases), diplopia (one case), worsening of general condition (one case), and malignancy screening (one case). In three cases, IOMs infiltrated the cavernous sinus, and one of them caused diplopia, while the other two caused restriction of gaze.

\section{DISCUSSION}

About $68 \%$ of PEMs affect the calvarium. These types of meningiomas are called intraosseous, calvarial, or intradiploic meningiomas (17). Hoye et al. described ectopic meningiomas as tumors unrelated to any intracranial structure (10). Crawford et al. defined real primary $\mathrm{IOM}$ as a lesion which does not affect the underlying dura (6). However, IOMs could infiltrate the dura in due course of time $(14,16)$. Several cases have been defined in the literature, with no dural involvement radiologically, however, the involvement being evident pathologically (3). Locations and forms of PIMs and IOMs may help differentiate the two entities radiologically. In comparison to PIMs, IOMs have higher tendency to be located on a broader base in the calvarium. In addition, vast majority of IOMs exhibit bone expansion, contour irregularities in inner and/or 

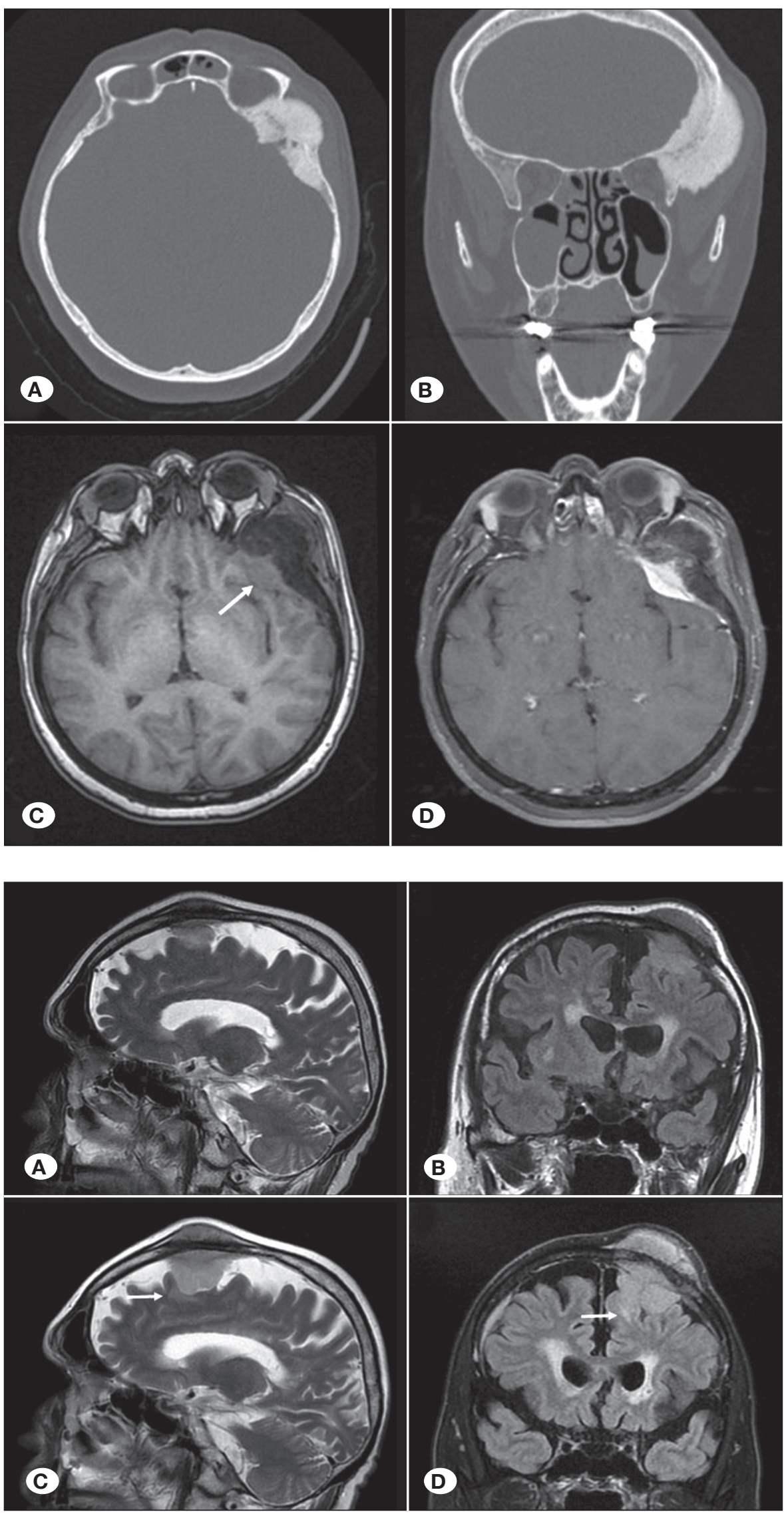

Figure 1: A 56-year-old woman with complaints of swelling on the head. A) Axial, B) coronal reformatted CT images show hyperostotic IOM, with radial bone spiculation in the left sphenoid ridge and calvarial bones. C) T1-weighted MR image shows hypointensity in the bone and soft tissue components (arrow). D) Contrastenhanced T1-weighted MR image shows heterogeneous contrast enhancement in the affected bone as well as intense homogeneous contrast enhancement in the soft tissue components and infiltrated dura mater.
Figure 2: A 53-year-old male patient with swelling on his head, who was found to have mixed type calvarial IOM. MR images in 2015 A) Sagittal T2-weighted MRI. B) Coronal FLAIR sequence. On MR images in 2017, it was observed that the mass increased in size and compressed the adjacent cerebral parenchyma, causing slight peritumoral edema, C) Sagittal T2-weighted MRI and D) Coronal fatsuppressed FLAIR sequence. 
Table I: Demographic and Radiological Findings of Patients with Intraosseous Meningioma

\begin{tabular}{cccccccccc}
\hline No & G & Age & Localization & $\begin{array}{c}\text { WHO } \\
\text { Grade }\end{array}$ & CT Pattern & Lesion Contour & $\begin{array}{c}\text { Dural } \\
\text { Ca ++ }\end{array}$ & $\begin{array}{c}\text { Cerebral } \\
\text { Edema }\end{array}$ & $\begin{array}{c}\text { Follow-up } \\
\text { (months) }\end{array}$ \\
\hline I & M & 52 & Skull base & I & Hyperostotic & Irregularity & + & - & 16 \\
\hline II & F & 56 & SR + Cal & I & Hyperostotic & Radial bone spiculation & - & - & 1 \\
\hline III & F & 62 & SR + Cal & I & $\begin{array}{c}\text { Mildly } \\
\text { hyperostotic }\end{array}$ & Radial bone spiculation & + & - & 59 \\
\hline III & F & 62 & SR + Cal & I & Hyperostotic & Radial bone spiculation & - & $-/+$ & 59 \\
\hline IV & F & 46 & SR & I & Hyperostotic & Irregularity & + & ++ & 9 \\
\hline V & F & 47 & SR & I & Hyperostotic & Irregularity & - & + & 73 \\
\hline VI & F & 49 & SR & I & Hyperostotic & Radial bone spiculation & - & - & 19 \\
\hline VII & M & 53 & Cal convexity & I & Mixed & Radial bone spiculation & - & $-/+$ & 21 \\
\hline VIII & M & 55 & Skull base & I & Hyperostotic & Radial bone spiculation & - & ++ & 1 \\
\hline IX & F & 53 & SR + Cal & I & Hyperostotic & Radial bone spiculation & - & - & 9 \\
\hline X & M & 80 & SR + Cal & II & Mixed & Irregularity & - & ++ & 1 \\
\hline
\end{tabular}

G: Gender, F: Female, M: Male, Ca+': Calcification, SR: sphenoid ridge, Cal: Calvarium.
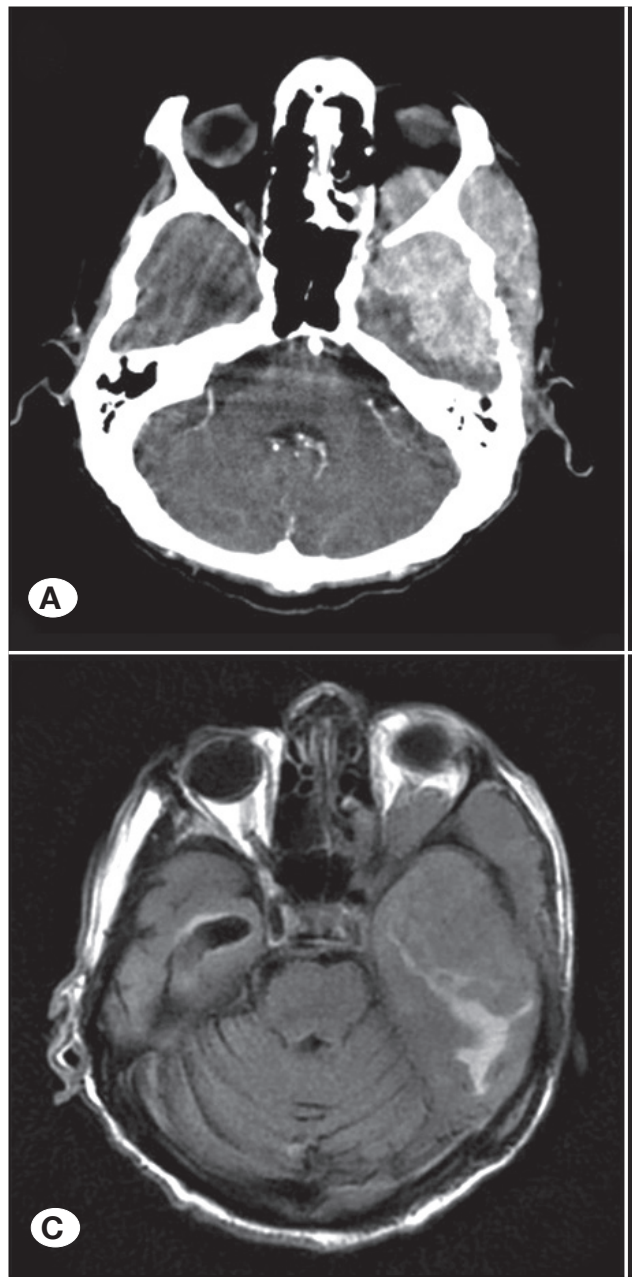

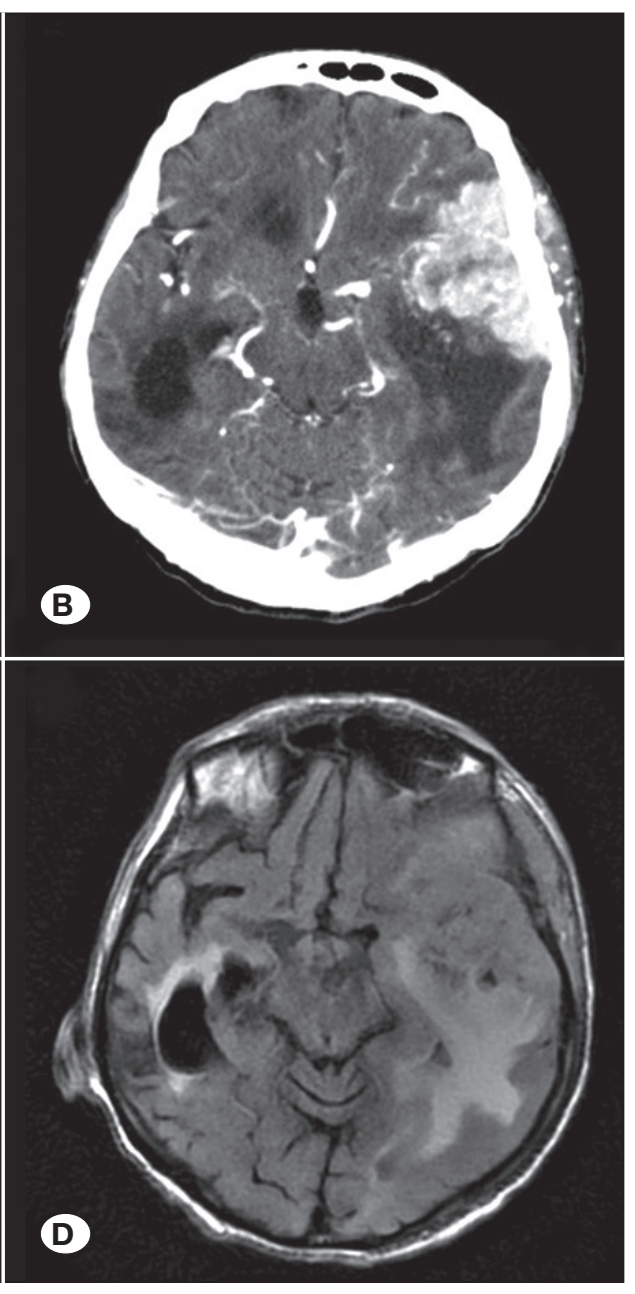

Figure 3: An 80-year-old male patient with $\mathrm{WHO}$ grade II IOM involving the left sphenoid ridge + calvarium, who had brain CT and MRI images due to impaired consciousness.

A, B) On contrast-enhanced CT images, intensely enhancing large soft tissue components of the mass extending to intraorbital space and intracranial and temporal fossa as well as causing prominent peritumoral brain edema were observed.

C, D) On Axial FLAIR sequence, soft tissue components of the mass, which were isointense to cerebral parenchyma and showed prominent peritumoral edema and right temporal periventricular interstitial edema, were observed. 

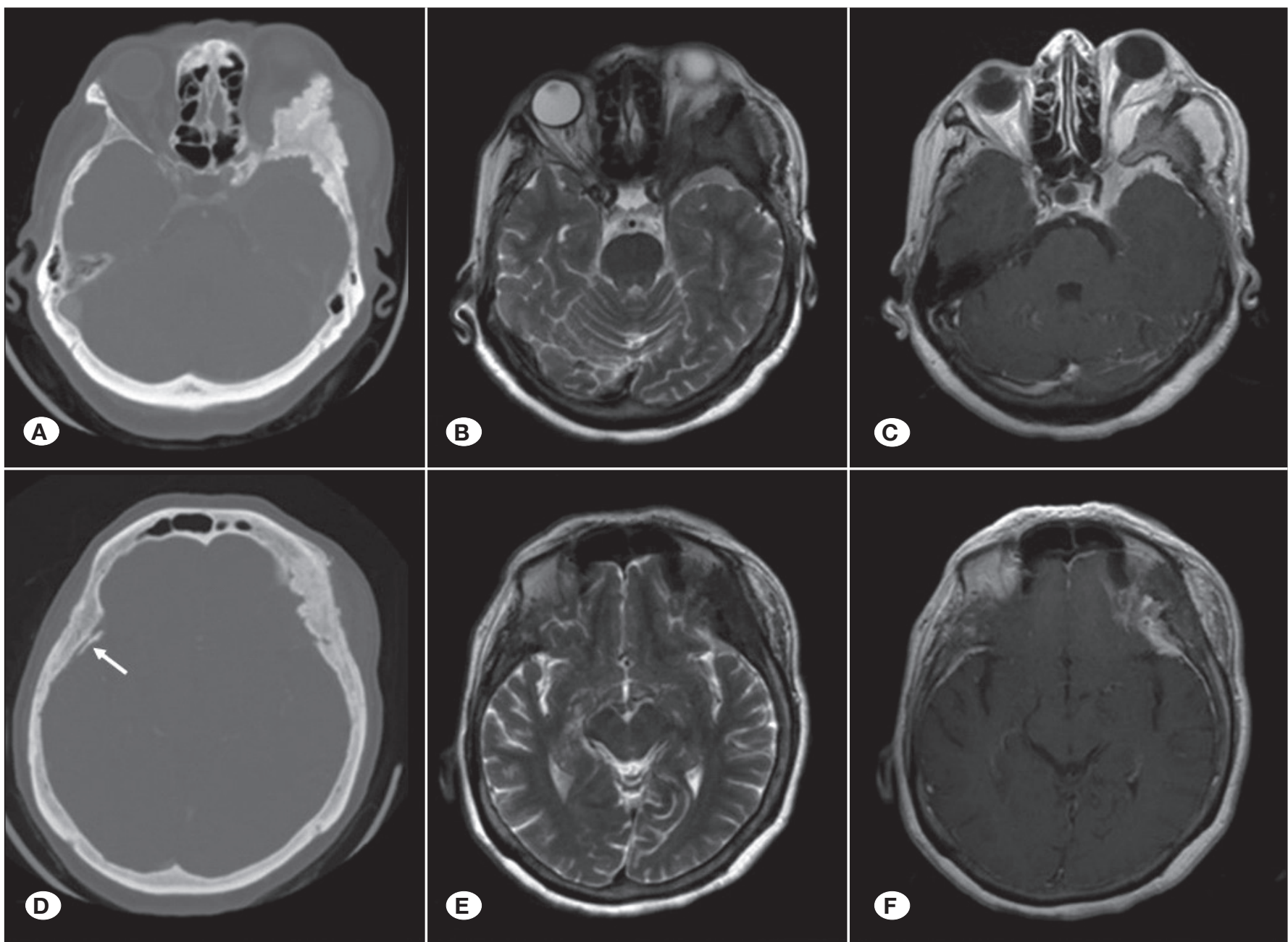

Figure 4: A 62-year-old female patient with left orbital swelling. A) CT image shows hyperostotic IOM with radial spicule contour in the left orbital walls and calvarial bones. Mild sclerotic IOM was observed in the right sphenoid ridge and calvarial bone. B) T2-weighted MR image shows IOM accompanied by soft tissue components, which led to exophthalmos in the left orbit. C) Contrast-enhanced T1weighted MR images show IOM with intraorbital, intracranial, and extracranial soft tissue components with intense contrast enhancement on the left. Contrast enhancement of an IOM in the sphenoid ridge and in the adjacent calvarial bone is observed on the right. D) CT image shows a linear dural calcification (arrow) accompanying the right IOM. E) T2-weighted MR image shows IOMs with bilateral hypointense signals. F) Contrast-enhanced T1-weighted MR image shows contrast enhancement in the affected bones and adjacent dura mater.

outer tabula, and accompanying soft tissue components (30). Based on commonly accepted classification in the literature, PEMs are classified as purely extracalvarial (Type I), purely calvarial (Type II), or calvarial with extracalvarial extension (type III) $(5,7,12,17,19)$. Lang et al. reviewed the literature and found that $32 \%$ of the cases in the literature can be classified as Type I, $45 \%$ as Type II, and $23 \%$ as type III tumors (17). Since the present study included the tumors localized only in the cranial bones (i.e., the skull base and convexity bones), Type I tumors were excluded. In the present study, all the IOMs were type III tumors, which reflects a higher frequency as compared to that mentioned in the present literature. IOMs could be localized in the sphenoid ridge, calvarial convexity or skull base bones, and more frequently in the periorbital area $(7,12,13,17,24)$. Frequency of IOMs in the sphenoid ridge was reported to be $53 \%-90 \%$; while, the frequency of those in the calvarial convexity was reported as $10 \%-33 \%$ and of those in the skull base as $14 \%(7,12,13,17,24)$. Likewise, most of the IOMs in the present study were localized in both the sphenoid ridge + calvarium and in the sphenoid ridge.

The pathogenesis of IOMs remains unclear. Several hypotheses have been proposed concerning the etiology of IOMs; such as the misplaced undifferentiated mesenchymal stem cells; undifferentiated arachnoid cap cells related to blood vessels or cranial nerves traversing the skull; meningocytes or arachnoid cap cells, which remained within the cranial sutures during molding of head at birth; trauma related fracture; and dedifferentiation of mature arachnoid cells $(20,26,29)$. Lang et al. reported in their review that $8 \%$ of the 168 IOMs they studied were related to the suture line (17). In another review 
by Crawford et al, $14 \%$ of the IOM cases were linked to a history of trauma (6). In the present study, $63.6 \%$ of the IOMs had expansion into the suture lines, and the frequency of suture line association was higher than that has reported in the current literature. On the other hand, none of the patients in the present study had a history of trauma.

On radiography or CT examinations, the IOMs exhibited hyperostotic, mixed, or lytic patterns in the cranial bones (6). Invasion of the haversian canals by meningiomatous cells and their stimulation to an osteoblastic activity leads to hyperostosis $(1,4,12,13,25,29)$. As a result of hyperostosis, focal thickening develops on the bone, manifested as increased density on the radiographies or CT (5). Hyperostotic lesions secondary to IOMs were reported to be $60 \%-68 \%$ in radiography or CT studies $(1,6,12)$. In the present study, IOMs with hyperostotic pattern constituted $81.8 \%$ of all the lesions, which was relatively high compared to that mentioned in the literature. Fibrous dysplasia, osteoma, Paget's disease, PIMs with an overlying hyperostosis (with or without true bone involvement), metastatic cancer, osteosarcoma, as well as endocrine and metabolic derangements such as hyperparathyroidism, hypervitaminosis A or D, and inflammatory bone lesions should be considered in the differential diagnosis of a hyperostotic lesion of the cranial bones $(4-7,30)$. Detailed clinical and radiological examinations could narrow down the differential diagnosis. Furthermore, age of the patient can help distinguish fibrous dysplasia from intraosseous meningioma, since fibrous dysplasia generally ceases to grow after puberty, while IOMs mostly appear and continue to grow slowly after puberty. Besides, the tumor blush on angiography, which is a characteristic of meningiomas, is not observed in fibrous dysplasia $(4,7)$. Both fibrous dysplasia and IOMs can lead to osseous expansions, but these lesions can be differentiated by their osseous contours. Cortical table irregularities are observed in IOMs; whereas, the inner and outer tables are smoothly contoured in fibrous dysplasia (12). In meningioma en plaque, considerable hyperostosis may be observed in the overlying bone, and tumor enhancement may be mistaken for an overlying IOM with dural reaction.

Intraosseous meningioma has a characteristic MRI appearance of homogeneously dense tumor. Moreover, gadolinium enhancement within the skull may help differentiate meningioma en plaque and other osteoblastic skull lesions such as osteoma (non-enhancing), osteosarcoma (irregular contours, heterogeneous signal, and enhancement), and Paget's disease (heterogeneous signal and non-enhancing) (7). Additionally, laboratory tests such as serum alkaline phosphatase, parathormone, and vitamin A and D levels can help narrow down the differential diagnosis (7).

The incidence of mixed pattern IOMs in the literature has been reported to be $5 \%-35.7 \%(1,6,12)$. Mixed pattern was observed in $18.1 \%$ of the cases in the present study. In the differential diagnosis of mixed pattern IOMs, which show hyperostotic pattern, fibrous dysplasia, Paget's disease, metastases, osteosarcoma, endocrine, and metabolic diseases must be considered. Frequency of IOMs with osteolytic pattern was reported to be $31 \%$ by Crawford et al. and $8 \%$ by
Ilica et al. $(6,12)$. Since the study by Crawford et al. was only radiographic, inner structures of the lesions might not have been examined in as detail as with CT, and the frequency of the lytic pattern might have been higher compared to that of the mixed pattern (6). Arana et al. reported no IOMs with pure Iytic pattern (1). Similarly, no IOMs with pure Iytic pattern were observed in the present study. In the differential diagnosis of IOMs with osteolytic pattern, benign or malignant pathologies, which could cause osteolytic lesions in the cranial bones, should be considered $(5,6,16,23,26)$. Malignant lesions such as metastases, chondrosarcoma, and osteosarcoma are often indicated by clinical information about the known primary cancer, a rapidly enlarging skull lesion, or by radiographic features such as multiple lesions and ragged margins without sclerosis (7).

Linear dural calcifications in the adjacent dura may accompany IOMs $(11,12)$. Ilica et al. mentioned that superficial irregularity, expansion, and accompanying subdural calcification in the involved bone on CT are pathognomonic for the diagnosis of IOMs. The authors detected dural calcification accompanying IOMs in 7 of 53 cases (12). In the present study, dural calcification was detected in three cases. Furthermore, five of the IOMs in the study by llica et al. (12) were graded as WHO grade I and two as WHO grade II; whereas, in the present study all IOMs with dural calcification were grade I tumors.

IOMs tend to cause expansion of the affected bones. Ilica et al. reported expansion of the affected bones in $67.7 \%$ of cases. Furthermore, they reported that $62.5 \%$ of WHO grade I IOMs and all WHO grade II and III tumors exhibited expansion of the bones (12). On the other hand, in the present study, expansion of the affected bone was observed in $90.9 \%$ of the IOMs. However, all lesions leading to expansion in the present study were WHO grade $\mathrm{I}$.

MRI findings of the IOMs are similar to those of the intradural lesions and allow better delineation of the tumors that have extracalvarial soft tissue extension. IOMs are typically hypointense on T1-weighted images and hyperintense on T2-weighted images. A typical prominent and homogenous contrast enhancement is obtained after gadolinium administration. Dural tail, often found with intradural meningiomas, is not displayed by IOMs; however, gadolinium enhancement of the underlying dura may be prominent. The mentioned dural enhancement could be due to dural irritation or tumor invasion $(5,7)$. A widely varying rate of dural infiltration, in the range of $26 \%-87.5 \%$, was reported histopathologically for the IOMs in the literature $(13,22)$. On the other hand, radiographically, the infiltration rates of the IOMs were reported as $60.0 \%-68.8 \%$ $(3,12,17)$. There is no certain criterion for dural infiltration. Ilica et al. considered linear contrast enhancement no more than 3 $\mathrm{mm}$ in dura as dural contrast enhancement only; while, they classified linear or nodular contrast enhancement larger than $3 \mathrm{~mm}$ as tumor involvement (12). Bassouni et al. found dural enhancement in 11 out of 16 patients (68.8\%) on MRI and attributed it to dural infiltration (3). Nevertheless, the same authors found that, based on the histopathological examination carried out due to intraoperative suspicion of dural infiltration, two cases concluded not to have infiltration based on radio- 
logical examination, in fact, had dural infiltration. Bassouni et al. found that recurrence was observed in two cases that were not considered intraoperatively to have dural infiltration and suggested that adjacent dura should be removed along with the involved bone to prevent recurrence (3). In the present study, nine IOMs had 4-26-mm-thick soft tissue components associated with the dural surface, suggesting dural infiltration on contrast-enhanced MRI.

Peritumoral brain edema was reported in $38 \%-67 \%$ of the patients with a PIM on diagnostic CT or MRI scans (18). It is an important prognostic imaging finding in preoperative period and is responsible for clinical deterioration, depending upon the parenchymal involvement. Peritumoral brain edema increases the risk of postoperative complications, since it is associated with invasion of the arachnoid membrane (12). However, there are not enough studies in the literature on peritumoral brain edema secondary to IOMs. Using MR examination, llica et al. found peritumoral brain edema in $62.7 \%$ of IOMs (12). In the same study, $54.7 \%$ of WHO grade I IOMs and all grade II and grade III IOMs had peritumoral edema. However, no information was provided by the authors regarding the obliteration of the subarachnoid space and involvement of the cerebral parenchyma. On the other hand, in the present study, peritumoral edema was observed in six IOMs, two of which did not have edema on initial imaging studies, but developed it during the follow-up period (Figure $2 A-D)$. Five of the six IOMs which had peritumoral cerebral edema were WHO grade I, while one was WHO grade II. It was also revealed that the IOMs with no peritumoral cerebral edema evident on initial imaging studies, increase in size, with development of cerebral edema due to narrowing of the subarachnoid space and secondarily involvement of the cerebral parenchyma.

Irregularities or radial bone spiculation could be observed in inner, outer, or both the tabula contours of the affected cranial bones with IOMs $(7,12)$. Contour irregularity or radial bone spiculation would allow exclusion of fibrous dysplasia, but may not help in ruling out malignancies such as osteosarcoma or metastases $(2,7,12)$. In the literature, the frequencies of radial bone spiculation and cortical irregularity in IOMs are reported in the range of $4.6 \%-64.3 \%$ and $35.7 \%-47.7 \%$, respectively $(1,12)$. In our study, $60 \%$ of the IOMs had radial bone speculation, while $40 \%$ had cortical irregularity.

The WHO classifies meningiomas into three groups: WHO I is a typical or benign lesion (88\%-94\%), WHO II is atypical lesion (5\%-7\%), while WHO III is an anaplastic or malignant lesion (1\%-2\%) (9). Although IOMs are defined as slow growing, histologically benign lesions, there are studies in the literature reporting higher incidence $(13.8 \%-26.0 \%)$ of histopathologically atypical or malign subtypes $(5,12,17)$. Compared to the literature, a lower incidence rate of atypical IOMs was found $(9.1 \%)$ in the present study.

Multiple IOMs are quite rare. Only four cases with multiple IOMs have been reported in the literature $(8,22,28)$. While El Mahou et al. (8) found multiple IOMs involving the right sphenoid ridge and left parietal bone and Nakao et al. (22) in the bilateral parietal bones, multiple IOMs affecting the bilateral sphenoid ridge + calvarial bones were found in the present study (Figure 4A-F).

Clinical features of IOMs are not specific. Symptoms could vary depending upon location and size of the tumor and involvement of the adjacent structures. Most IOMs exist as painless expanding masses without abnormal neurological manifestations (32)). In four patients, expansion, exophthalmos, and orbital swelling associated with soft tissue components were observed in the affected bone. Two patients had diplopia and restriction of gaze due to infiltration of the cavernous sinus by IOM.

For the treatment of IOMs, total resection of the tumor along with the neighboring dura, if possible, has been mentioned to be curative $(3,5)$. When lesions in the skull base cannot be removed completely, surgery is performed to decompress the vital neural structures (5). Serial imaging should be used to follow the tumors that cannot be completely excised when they are histologically benign and neurologically asymptomatic (5). Unresectable benign IOMs causing neurologic deficits and atypical or malignant IOMs should be treated using adjuvant therapies such as radiotherapy, chemotherapy, or hormonal therapy (5).

The present study had several limitations. Firstly, the operative reports did not always specify whether the bone specimens included a dural component. Therefore, dural thickening and enhancement observed on post-contrast MR images were considered as dural involvement. Secondly, asymptomatic, small sized IOMs, with pure lytic pattern on CT scans, could have been mistaken by indentation of arachnoid granulation. And lastly, one case had contrast-enhanced CT scan, but no contrast-enhanced MRI examination, and in an another case, $\mathrm{CT}$ and MRI examinations were carried out without contrast medium.

\section{CONCLUSION}

IOMs are predominantly WHO grade I tumors, frequently located in the periorbital region and might cause brain edema. Vast majority of them exhibit hyperostotic pattern and lead to bone expansion and contour irregularities, mostly in the form of radial bone spiculation. They are frequently accompanied with dural infiltrations and soft tissue components extending out from the affected bone. Although IOMs are generally unilaterally localized, they may quite occasionally be seen bilaterally.

\section{REFERENCES}

1. Arana E, Diaz C, Latorre FF, Menor F, Revert A, Beltrán A, Navarro M: Primary intraosseous meningiomas. Acta Radiol 37:937-942, 1996

2. Asil K, Aksoy YE, Yaldiz C, Kahyaglu Z: Primary intraosseous meningioma mimicking osteosarcoma: Case report. Turk Neurosurg 25:174-176, 2015

3. Bassiouni H, Asgari S, Hübschen U, König HJ, Stolke D: Dural involvement in primary extradural meningiomas of the cranial vault. J Neurosurg 105:51-59, 2006 
4. Changhong L, Naiyin C, Yuehuan G, Lianzhong Z: Primary intraosseous meningiomas of the skull. Clin Radiol 52:546550, 1997

5. Chen TC: Primary intraosseous meningioma. Neurosurg Clin N Am 27:189-193, 2016

6. Crawford TS, Kliensxhmidt-Demasters BK, Lillehei KO: Primary intraosseous meningioma. Case report. J Neurosurg 83:912-915, 1995

7. Elder JB, Atkinson R, Zee C-S, Chen TC: Primary intraosseous meningioma. Neurosurg Focus 23:1-9, 2007

8. El Mahou S, Popa L, Constantin A, Jamard B, Cantagrel A, Mazieres $\mathrm{B}$, Laroche $\mathrm{M}$ : Multiple intraosseous meningiomas. Clin Rheumatol 25:553-554, 2006

9. Hale AT, Wang L, Strother MK, Chambless LB: Differentiating meningioma grade by imaging features on magnetic resonance imaging. J Clin Neurosci 48:71-75, 2018

10. Hoye SJ, Hoar CS Jr, Murray JE: Extracranial meningioma presenting as a tumor of the neck. Am J Surg 100:486-489, 1960

11. Hussaini SM, Dziurzynski K, Fratkin JD, Jordan JR, Hussain SA, Khan M: Intraosseous meningioma of the sphenoid bone. Radiol Case Rep 5:357, 2010

12. Ilica AT, Mossa-Basha M, Zan E, Vikani A, Pillai JJ, Gujar $\mathrm{S}$, Aygun N, Izbudak I: Cranial intraosseous meningioma: Spectrum of neuroimaging findings with respect to histopathological grades in 65 patients. Clin Imaging 38:599604, 2014

13. Kim KS, Rogers LF, Goldblatt D: CT features of hyperostosing meningioma en plaque. AJR Am J Roentgenol 149:10171023, 1987

14. Kudo H, Maeda S, Takamoto T, Tamaki N: Intraosseous meningioma with a dural defect. Neurol Med Chir (Tokyo) 38:229-231, 1998

15. Kunimatsu A, Kunimatsu N, Kamiya K, Katsura M, Mori H, Ohtomo K: Variants of meningiomas: A review of imaging findings and clinical features. Jpn J Radiol 34:459-469, 2016

16. Kwon SY, Shin HS, Kim TH, Kim HJ: Primary intraosseous osteolytic meningioma of the skull mimicking scalp mass: A case report and review of literature. Brain Tumor Res Treat 3:151-155, 2015

17. Lang FF, Macdonald OK, Fuller GN, DeMonte F: Primary extradural meningiomas: A report on nine cases and review of the literature from the era of computerized tomography scanning. J Neurosurg 93:940-950, 2000

18. Latini F, Larsson EM, Ryttlefors M: Rapid and accurate MRI segmentation of peritumoral brain edema in meningiomas. Clin Neuroradiol 27:145-152, 2017
19. Matschke J, Addo J, Bernreuther C, Zustin J: Osseous changes in meningioma en plaque. Anticancer Res 31:591596, 2011

20. Mattox A, Hughes B, Oleson J, Reardon D, McLendon $\mathrm{R}$, Adamson C: Treatment recommendations for primary extradural meningiomas. Cancer 117:24-38, 2011

21. McGuire TP, Palme CE, Perez-Ordonez B, Gilbert RW, Sándor GK: Primary intraosseous meningioma of the calvaria: Analysis of the literature and case report. Oral Surg Oral Med Oral Pathol Oral Radiol Endod 104:e34-41, 2007

22. Nakao N, Kubo K, Moriwaki H: Multiple growths of primary calvarial meningiomas. Neurosurgery 29:452-455, 1991

23. Rosahl SK, Mirzayan MJ, Samii M: Osteolytic intra-osseous meningiomas: Illustrated review. Acta Neurochir (Wien) 146:1245-1249, 2004

24. Shrivastava RK, Sen C, Costantino PD, Della Rocca R: Sphenoorbital meningiomas: Surgical limitations and lessons learned in their long-term management. J Neurosurg 103:491497, 2005

25. Talacchi A, Corsini F, Gerosa M: Hyperostosing meningiomas of the cranial vault with and without tumor mass. Acta Neurochir 153:53-61, 2011

26. Tokgoz N, Oner YA, Kaymaz M, Ucar M, Yilmaz G, Tali TE: Primary intraosseous meningioma: CT and MRI appearance. AJNR Am J Neuroradiol 26:2053-2056, 2005

27. Verma SK, Satyarthee G, Borkar SA, Singh M, Sharma BS: Orbital roof intradiploic meningioma in a 16-year-old girl. J Pediatr Neurosci 10:51-54, 2015

28. Wagman AD, Weiss EK, Riggs HE: Hyperplasia of the skull associated with intraosseous meningioma in the absence of gross tumor. J Neuropathol Exp Neural 19:111-115, 1960

29. Yamamoto J, Kurokawa T, Miyaoka R, Soejima Y, Nishizawa S: Primary intraosseous meningioma in the calvaria: Morphological feature changes on magnetic resonance images over several years. Jpn J Radiol 33:437-440, 2015

30. Yun JH, Lee SK: Primary osteolytic intraosseous atypical meningioma with soft tissue and dural invasion: Report of a case and review of literatures. J Korean Neurosurg Soc 56:509-512, 2014

31. Zakhari N, Torres C, Castillo M, Nguyen TB: Uncommon cranial meningioma: Key imaging features on conventional and advanced imaging. Clin Neuroradiol 27:135-144, 2017

32. Zhang S, Zhang J, Chen J, Ai X, He X: Frontal intradiploic meningioma with progressive intracranial invasion: $A$ rare case report. Medicine (Baltimore) 96:e7903, 2017 